Proyecciones Journal of Mathematics

Vol. 34, No 4, pp. 359-375, December 2015.

Universidad Católica del Norte

Antofagasta - Chile

\title{
On the hyperstability of a Cauchy-Jensen type functional equation in Banach spaces
}

\author{
Iz-iddine EL-Fassi \\ and \\ Samir Kabbaj \\ University of Ibn Tofail, Morocco \\ Received : June 2015. Accepted : October 2015
}

\begin{abstract}
In this paper, we establish some hyperstability results of the following Cauchy-Jensen functional equation$$
f\left(\frac{x+y}{2}+z\right)+f\left(\frac{x-y}{2}+z\right)=f(x)+2 f(z)
$$

in Banach spaces.
\end{abstract}

Subjclass [2010] : Primary 39B82, 39B62; Secondary 47H14, 47H10.

Keywords : Hyperstability, Cauchy-Jensen, fixed point theorem. 


\section{Introduction and Preliminaries}

The stability problem of functional equations was posed for the first time by Ulam [27] in the year 1940. Ulam stated the problem as follows:

Let $(G,$.$) be a group and let (H, ., d)$ be a metric group with the metric $d$. Given $\delta>0$, does there exist $\epsilon>0$ such that if a mapping $h: G \rightarrow H$ satisfies the inequality

$$
d(h(x y), h(x) h(y)) \leq \delta
$$

for all $x, y \in G$, then there is a homomorphism $a: G \rightarrow H$ with

$$
d(h(x), a(x)) \leq \epsilon
$$

for all $x \in G$ ?

Ulam's problem was partially solved by Hyers in 1941 in the context of Banach spaces with $\epsilon=\delta$ as shown below [14].

Theorem 1.1. [D. H. Hyers (1941)] Let $E$ be a normed vector space, $F$ a Banach space and suppose that the mapping $f: E \rightarrow F$ satisfies the inequality

$$
\|f(x+y)-f(x)-f(y)\| \leq \epsilon,
$$

for all $x, y \in E$ where $\epsilon$ is a constant. Then the limit

$$
T(x)=\lim _{n \rightarrow \infty} 2^{-n} f\left(2^{n} x\right)
$$

exists for each $x \in E$ and $T$ is the unique additive mapping satisfying

$$
\|f(x)-T(x)\| \leq \epsilon
$$

for all $x \in E$. Also, if for each $x$ the function $t \rightarrow f(t x)$ from $\mathbf{R}$ to $F$ is continuous for each fixed $x$, then $T$ is linear. If $\mathrm{f}$ is continuous at a single point of $E$, then $T$ is continuous in $E$.

Aoki [1], Z. Gajda [11] and Th. M. Rassias [23] provided a generalization of the Hyers theorem for additive and linear mappings, respectively, by allowing the Cauchy difference to be unbounded.

Theorem 1.2. Let $f: E \rightarrow F$ be a mapping from a real normed vector space $E$ into a Banach space $F$ satisfying the inequality 


$$
\|f(x+y)-f(x)-f(y)\| \leq \theta\left(\|x\|^{p}+\|y\|^{p}\right),
$$

for all $x, y \in E \backslash\{0\}$, where $\theta$ and $p$ are constants with $\theta>0$ and $p \neq 1$. Then there exists a unique additive mapping $T: E \rightarrow F$ such that

$$
\|f(x)-T(x)\| \leq \frac{\theta}{\left|1-2^{p-1}\right|}\|x\|^{p},
$$

for all $x \in E \backslash\{0\}$.

Theorem 1.2 is due to Aoki [1] for $0<p<1$ (see also [23]; Gajda [11] for $p>1$; Hyers [14] for $p=0$ and Th. M. Rassias [24] for $p<0$ (see [[25], page 326] and [5]).

Beginning around the year 1980 the topic of approximate homomorphisms, or the stability of the equation of homomorphism, was studied by a number of mathematicians (see 10, 16, 17, 18, 26, 12, 15, 20, 21).

Recently, interesting results concerning Cauchy-Jensen functional equation

$$
f\left(\frac{x+y}{2}+z\right)+f\left(\frac{x-y}{2}+z\right)=f(x)+2 f(z),
$$

have been obtained in [2] and [20].

Lemma 1.1. Let $X$ and $Y$ be vector spaces. If a mapping $f: X \rightarrow Y$ satisfies (1.3), then the mapping $f: X \rightarrow Y$ is Cauchy additive and $f$ is a solution of the Jensen equation $2 f\left(\frac{x+y}{2}\right)=f(x)+f(y)$.

We say a functional equation $D$ is hyperstable if any function $f$ satisfying the equation $D$ approximately is a true solution of $D$. The term hyperstability was used for first time probably in [19]. However, it seems that the first hyperstability result was published in [4] and concerned the ring homomorphisms. The hyperstability results for Cauchy equation were investigated by Brzdek in [7, 8, 9]. Gselmann in [13] studied the hyperstability of the parametric fundamental equation of information. In [3] Bahyrycz and Piszczek provided the hyperstability of the Jensen functional equation.

In this paper, we present the hyperstability results for the CauchyJensen functional equation (1.3) in Banach spaces.

The method of the proof of the main results is motivated by an idea used by Brzdek in [7, 8, 9] and further by Piszczek in [22]. It is based on 
a fixed point theorem for functional spaces obtained by Brzdek et al. (see [[6], Theorem 1]).

Throughout the paper, $\mathbf{N}, \mathbf{R}, \mathbf{R}_{+}$and $\mathbf{N}_{m_{0}}$ denote the set of all positive integers, the set of real numbers, the set of positive real numbers and the set of all integers greater than or equal to $m_{0}$, respectively.

First, we take the following three hypotheses (all notations come from [6]).

(H1) $X$ is a nonempty set, $Y$ is a Banach space, $f_{1}, \ldots f_{k}: X \rightarrow X$ and $L_{1}, \ldots . L_{k}: X \rightarrow \mathbf{R}_{+}$are given.

(H2) $\mathcal{T}: Y^{X} \rightarrow Y^{X}$ is an operator satisfying the inequality

$$
\|\mathcal{T} \xi(x)-\mathcal{T} \mu(x)\| \leq \sum_{i=1}^{k} L_{i}(x)\left\|\xi\left(f_{i}(x)\right)-\mu\left(f_{i}(x)\right)\right\|
$$

for all $\xi, \mu \in Y^{X}, x \in X$.

(H3) $\Lambda: \mathbf{R}_{+}{ }^{X} \rightarrow \mathbf{R}_{+}{ }^{X}$ is a linear operator defined by

$$
\Lambda \delta(x):=\sum_{i=1}^{k} L_{i}(x) \delta\left(f_{i}(x)\right)
$$

for all $\delta \in \mathbf{R}_{+}{ }^{X}, x \in X$.

The mentioned fixed point theorem is stated as follows.

Theorem 1.3. Let hypotheses (H1)-(H3) be valid and functions $\varepsilon: X \rightarrow$ $\mathbf{R}_{+}$and let $\varphi: X \rightarrow Y$ fulfil the following two conditions:

$$
\begin{gathered}
\|\mathcal{T} \varphi(x)-\varphi(x)\| \leq \varepsilon(x), \quad x \in X \\
\varepsilon^{*}(x):=\sum_{n=0}^{\infty} \Lambda^{n} \varepsilon(x)<\infty, \quad x \in X .
\end{gathered}
$$


Then, there exists a unique fixed point $\psi$ of $\mathcal{T}$ with

$$
\|\varphi(x)-\psi(x)\| \leq \varepsilon^{*}(x), \quad x \in X .
$$

Moreover

$$
\psi(x)=\lim _{n \rightarrow \infty} \mathcal{T}^{n} \varphi(x), \quad x \in X
$$

\section{Hyperstability Results of eq (1.3)}

The following theorems are the main results in this paper and concern the hyperstability of equation (1.3). Moreover the results that we will obtained in these theorems corresponds the results obtained in $[3,9]$.

Theorem 2.1. Let $X$ be a normed space, $Y$ be a Banach space, $c \geq 0$ and $p, q, r \in \mathbf{R}$ such that $p+q+r<0$. If $f: X \rightarrow Y$ satisfies

$$
\left\|f\left(\frac{x+y}{2}+z\right)+f\left(\frac{x-y}{2}+z\right)-2 f(z)-f(x)\right\| \leq c\|x\|^{p}\|y\|^{q}\|z\|^{r}
$$

for all $x, y, z \in X \backslash\{0\}$, then $f$ is a solution of (1.3) on $X \backslash\{0\}$.

Proof. It is sufficient to consider only the case $p+q<0$. Replacing $(x, y, z)$ by $(m x, m x, m)$, with $m \in \mathbf{N}_{1}$, in (2.1), we get

$$
\|f((1+m) x)-f(m x)-f(x)\| \leq c m^{p+q}\|x\|^{p+q+r}
$$

for all $x \in X \backslash\{0\}$. Further put

$$
\begin{aligned}
\mathcal{T} \xi(x):= & \xi((1+m) x)-\xi(m x), x \in X \backslash\{0\}, \xi \in Y^{X \backslash\{0\}}, \\
& \varepsilon(x):=c m^{p+q}\|x\|^{p+q+r}, x \in X \backslash\{0\} .
\end{aligned}
$$

Then the inequality (2.2) takes the form

$$
\|\mathcal{T} f(x)-f(x)\| \leq \varepsilon(x), \quad x \in X \backslash\{0\} .
$$

The operator

$$
\Lambda \delta(x):=\delta((1+m) x)+\delta(m x), \quad x \in X \backslash\{0\}, \quad \delta \in \mathbf{R}_{+}^{X \backslash\{0\}}
$$

has the form described in (H3) with $k=2$ and $f_{1}(x)=(1+m) x, f_{2}(x)=$ $m x$, and $L_{1}(x)=L_{2}(x)=1$ for $x \in X \backslash\{0\}$. 
Moreover, for every $\xi, \mu \in Y^{X \backslash\{0\}}$ and $x \in X \backslash\{0\}$, we obtain

$$
\begin{array}{r}
\|\mathcal{T} \xi(x)-\mathcal{T} \mu(x)\|=\left\|(\xi-\mu)\left(f_{1}(x)\right)-(\xi-\mu)\left(f_{2}(x)\right)\right\| \\
\leq\left\|(\xi-\mu)\left(f_{1}(x)\right)\right\|+\left\|(\xi-\mu)\left(f_{2}(x)\right)\right\| \\
=\sum_{i=1}^{2} L_{i}(x)\left\|(\xi-\mu)\left(f_{i}(x)\right)\right\| .
\end{array}
$$

So, (H2) is valid. Next, we can find $m_{0} \in \mathbf{N}_{1}$ such that

$$
(1+m)^{p+q+r}+m^{p+q+r}<1 \text { for all } m \geq m_{0} .
$$

Therefore, we obtain that

$$
\begin{aligned}
& \varepsilon^{*}(x):=\sum_{n=0}^{\infty} \Lambda^{n} \varepsilon(x) \\
& =c m^{p+q}\|x\|^{p+q+r} \sum_{n=0}^{\infty}\left((1+m)^{p+q+r}+m^{p+q+r}\right)^{n} \\
& =\frac{c m^{p+q}\|x\|^{p+q+r}}{1-(1+m)^{p+q+r}-m^{p+q+r}}, x \in X \backslash\{0\}, m \geq m_{0}
\end{aligned}
$$

Thus, according to Theorem 1.3, for each $m \geq m_{0}$ there exists a unique solution $\mathrm{F}_{m}: X \backslash\{0\} \rightarrow Y$ of the equation

$$
F_{m}(x)=F_{m}((1+m) x)-F_{m}(m x)
$$

for all $x \in X \backslash\{0\}$, such that

$$
\left\|f(x)-F_{m}(x)\right\| \leq \frac{c m^{p+q}\|x\|^{p+q+r}}{1-(1+m)^{p+q+r}-m^{p+q+r}},
$$

for all $x \in X \backslash\{0\}, \quad m \geq m_{0}$.

Moreover

$$
F_{m}(x)=\lim _{n \rightarrow \infty} \mathcal{T}^{n} f(x)
$$

We show that

$$
\begin{gathered}
\left\|\mathcal{T}^{n} f\left(\frac{x+y}{2}+z\right)+\mathcal{T}^{n} f\left(\frac{x-y}{2}+z\right)-2 \mathcal{T}^{n} f(z)-\mathcal{T}^{n} f(x)\right\| \leq \\
c\left((1+m)^{p+q+r}+m^{p+q+r}\right)^{n}\|x\|^{p}\|y\|^{q}\|z\|^{r}
\end{gathered}
$$

for all $x, y, z \in X \backslash\{0\}$ and $n \in \mathbf{N}$. 
Indeed, if $n=0$, then (2.3) is simply (2.1). So, fix $n \in \mathbf{N}$ and suppose that (2.3) holds for $n$. Then

$$
\begin{aligned}
& \left\|\mathcal{T}^{n+1} f\left(\frac{x+y}{2}+z\right)+\mathcal{T}^{n+1} f\left(\frac{x-y}{2}+z\right)-2 \mathcal{T}^{n+1} f(z)-\mathcal{T}^{n+1} f(x)\right\|= \\
& \| \mathcal{T}^{n} f\left((1+m)\left(\frac{x+y}{2}+z\right)\right)-\mathcal{T}^{n} f\left(m\left(\frac{x+y}{2}+z\right)\right) \\
& +\mathcal{T}^{n} f(1+m)\left(\frac{x-y}{2}+z\right)-\mathcal{T}^{n} f\left(m\left(\frac{x-y}{2}+z\right)\right) \\
& -2 \mathcal{T}^{n} f((m+1) z)+2 \mathcal{T}^{n} f(m z)-\mathcal{T}^{n} f((m+1) x)+\mathcal{T}^{n} f(m x) \| \\
& \leq \| \mathcal{T}^{n} f\left((m+1)\left(\frac{x+y}{2}+z\right)\right)+\mathcal{T}^{n} f\left((m+1)\left(\frac{x-y}{2}+z\right)\right) \\
& -2 \mathcal{T}^{n} f((1+m) z)-\mathcal{T}^{n} f(1+m) x \| \\
& +\left\|\mathcal{T}^{n} f\left(m\left(\frac{x+y}{2}+z\right)\right)+\mathcal{T}^{n} f\left(m\left(\frac{x-y}{2}+z\right)\right)-2 \mathcal{T}^{n} f(m z)-\mathcal{T}^{n} f(m x)\right\| \\
& \leq c\left[(m+1)^{p+q+r}+m^{p+q+r}\right]^{n}\|(1+m) x\|^{p}\|(1+m) y\|^{q}\|(1+m) z\|^{r} \\
& +c\left[(1+m)^{p+q+r}+m^{p+q+r}\right]^{n}\|m x\|^{p}\|m y\|^{q}\|m z\|^{r} \\
& =c\left((1+m)^{p+q+r}+m^{p+q+r}\right)^{n+1}\|x\|^{p}\|y\|^{q}\|z\|^{r}
\end{aligned}
$$

for all $x, y, z \in X \backslash\{0\}$.

Thus, by induction, we have shown that (2.3) holds for all $x, y, z \in$ $X \backslash\{0\}$ and for all $n \in \mathbf{N}$. Letting $n \rightarrow \infty$ in (2.3), we obtain

$$
F_{m}\left(\frac{x+y}{2}+z\right)+F_{m}\left(\frac{x-y}{2}+z\right)=F_{m}(x)+2 F_{m}(z),
$$

for all $x, y, z \in X \backslash\{0\}$. So, we find a sequence $\left(F_{m}\right)_{m \geq m_{0}}$ satisfies (1.3) on $X \backslash\{0\}$ such that

$$
\left\|f(x)-F_{m}(x)\right\| \leq \frac{c m^{p+q}\|x\|^{p+q+r}}{1-(1+m)^{p+q+r}-m^{p+q+r}}, \quad x \in X \backslash\{0\}, \quad m \geq m_{0} .
$$

It follows, with $m \rightarrow \infty$, that $f$ is a solution of (1.3) on $X \backslash\{0\}$.

In a similar way we can prove the following three theorems.

Theorem 2.2. Let $X$ be a normed space, $Y$ be a Banach space, $c \geq 0$ and $p, q, r \in \mathbf{R}$ such that $p+q+r>1$. If $f: X \rightarrow Y$ satisfies (2.1) then $f$ is a solution of (1.3) on $X \backslash\{0\}$. 
Proof. Since $p+q+r>1$, one of $p, r+q$ must be positive. It is sufficient to consider only the case $q+r>0$ and $p \in \mathbf{R}$. Replacing $(x, y, z)$ by $\left(x, \frac{x}{m}, \frac{x}{m}\right)$, with $m \in \mathbf{N}_{1}$, in (2.1), we get

$$
\left\|f\left(\frac{3+m}{2 m} x\right)+f\left(\frac{1+m}{2 m} x\right)-2 f\left(\frac{x}{m}\right)-f(x)\right\| \leq \frac{c}{m^{q+r}}\|x\|^{p+q+r}
$$

for all $x \in X \backslash\{0\}$. Define operators $\mathcal{T}: Y^{X \backslash\{0\}} \rightarrow Y^{X \backslash\{0\}}$ and $\Lambda$ : $\mathbf{R}_{+} X \backslash\{0\} \rightarrow \mathbf{R}_{+} X \backslash\{0\}$ by

$$
\begin{gathered}
\mathcal{T} \xi(x):=\xi\left(\frac{3+m}{2 m} x\right)+\xi\left(\frac{1+m}{2 m} x\right)-2 \xi\left(\frac{x}{m}\right), x \in X \backslash\{0\}, \xi \in Y^{X \backslash\{0\}}, \\
\Lambda \delta(x):=\delta\left(\frac{3+m}{2 m} x\right)+\delta\left(\frac{1+m}{2 m} x\right)+2 \delta\left(\frac{x}{m}\right), \quad x \in X \backslash\{0\}, \delta \in \mathbf{R}_{+}{ }^{X \backslash\{0\}} .
\end{gathered}
$$

Then it is easily seen that $\Lambda$ has the form described in (H3) with $k=3$ and

$$
\begin{gathered}
f_{1}(x)=\frac{3+m}{2 m} x, \quad f_{2}(x)=\frac{1+m}{2 m} x, \quad f_{3}(x)=\frac{x}{m}, \quad L_{1}(x)=L_{2}(x)=1 \\
\text { and } L_{3}(x)=2, \quad x \in X \backslash\{0\} .
\end{gathered}
$$

Then the inequality (2.4) take the form

$$
\|\mathcal{T} f(x)-f(x)\| \leq \varepsilon(x), \quad x \in X \backslash\{0\},
$$

with

$$
\varepsilon(x):=\frac{c}{m^{q+r}}\|x\|^{p+q+r} .
$$

Moreover, for every $\xi, \mu \in Y^{X \backslash\{0\}}$ and $x \in X \backslash\{0\}$, we have $\|\mathcal{T} \xi(x)-\mathcal{T} \mu(x)\|=\left\|(\xi-\mu)\left(f_{1}(x)\right)+(\xi-\mu)\left(f_{2}(x)\right)-2(\xi-\mu)\left(f_{3}(x)\right)\right\|$

$\leq \sum_{i=1}^{3} L_{i}(x)\left\|(\xi-\mu)\left(f_{i}(x)\right)\right\|$.

So, (H2) is valid. We observe that there exists $m_{0} \in \mathbf{N}_{1}$ such that

$$
\left[\frac{3+m}{2 m}\right]^{p+q+r}+\left[\frac{1+m}{2 m}\right]^{p+q+r}+\frac{2}{m^{p+q+r}}<1 \text { for all } m \geq m_{0} .
$$

We have 


$$
\begin{aligned}
& \varepsilon^{*}(x): \\
& =\sum_{n=0}^{\infty} \Lambda^{n} \varepsilon(x) \\
& =\frac{c}{m^{q+r}}\|x\|^{p+q+r} \sum_{n=0}^{\infty}\left(\left[\frac{3+m}{2 m}\right]^{p+q+r}+\left[\frac{1+m}{2 m}\right]^{p+q+r}+\frac{2}{m^{p+q+r}}\right)^{n} \\
& =\frac{c\|x\|^{p+q+r}}{m^{q+r}\left(1-\left[\frac{3+m}{2 m}\right]^{p+q+r}-\left[\frac{1+m}{2 m}\right]^{p+q+r}-\frac{2}{m^{p+q+r}}\right)}
\end{aligned}
$$

for all $x \in X \backslash\{0\}$ and $m \geq m_{0}$.

Thus, according to Theorem 1.3, for each $m \geq m_{0}$ there exists a unique solution $\mathrm{F}_{m}: X \backslash\{0\} \rightarrow Y$ of the equation

$$
F_{m}(x)=F_{m}\left(\frac{3+m}{2 m} x\right)+F_{m}\left(\frac{1+m}{2 m} x\right)-2 F_{m}\left(\frac{x}{m}\right),
$$

for all $x \in X \backslash\{0\}$, such that

$$
\left\|f(x)-F_{m}(x)\right\| \leq \frac{c\|x\|^{p+q+r}}{m^{q+r}\left(1-\left[\frac{3+m}{2 m}\right]^{p+q+r}-\left[\frac{1+m}{2 m}\right]^{p+q+r}-\frac{2}{m^{p+q+r}}\right)},
$$

for all $x \in X \backslash\{0\}$ and $m \geq m_{0}$.

Moreover

$$
F_{m}(x)=\lim _{n \rightarrow \infty} \mathcal{T}^{n} f(x), \quad x \in X \backslash\{0\} .
$$

We show that

$$
\begin{gathered}
\left\|\mathcal{T}^{n} f\left(\frac{x+y}{2}+z\right)+\mathcal{T}^{n} f\left(\frac{x-y}{2}+z\right)-2 \mathcal{T}^{n} f(z)-\mathcal{T}^{n} f(x)\right\| \leq \\
(2.5) c\left(\left[\frac{3+m}{2 m}\right]^{p+q+r}+\left[\frac{1+m}{2 m}\right]^{p+q+r}+\frac{2}{m^{p+q+r}}\right)^{n}\|x\|^{p}\|y\|^{q}\|z\|^{r}
\end{gathered}
$$

for all $x, y, z \in X \backslash\{0\}$ and $n \in \mathbf{N}$.

Indeed, if $n=0$, then (2.3) is simply (2.1). So, fix $n \in \mathbf{N}$ and suppose that (2.3) holds for $n$. Then 


$$
\begin{aligned}
& \left\|\mathcal{T}^{n+1} f\left(\frac{x+y}{2}+z\right)+\mathcal{T}^{n+1} f\left(\frac{x-y}{2}+z\right)-2 \mathcal{T}^{n+1} f(z)-\mathcal{T}^{n+1} f(x)\right\| \leq \\
& \left\|\mathcal{T}^{n} f\left(\frac{3+m}{2 m}\left(\frac{x+y}{2}+z\right)\right)+\mathcal{T}^{n} f\left(\frac{3+m}{2 m}\left(\frac{x-y}{2}+z\right)\right)-2 \mathcal{T}^{n} f\left(\frac{3+m}{2 m} z\right)-\mathcal{T}^{n} f\left(\frac{3+m}{2 m} x\right)\right\| \\
& +\left\|\mathcal{T}^{n} f\left(\frac{1+m}{2 m}\left(\frac{x+y}{2}+z\right)\right)+\mathcal{T}^{n} f\left(\frac{1+m}{2 m}\left(\frac{x-y}{2}+z\right)\right)-2 \mathcal{T}^{n} f\left(\frac{1+m}{2 m} z\right)-\mathcal{T}^{n} f\left(\frac{1+m}{2 m} x\right)\right\| \\
& +\left\|2 \mathcal{T}^{n} f\left(\frac{1}{m}\left(\frac{x+y}{2}+z\right)\right)+\mathcal{T}^{n} f\left(\frac{1}{m}\left(\frac{x-y}{2}+z\right)\right)-2 \mathcal{T}^{n} f\left(\frac{z}{m}\right)-\mathcal{T}^{n} f\left(\frac{x}{m}\right)\right\| \\
& \leq c\left[\left(\frac{3+m}{2 m}\right)^{p+q+r}+\left(\frac{1+m}{2 m}\right)^{p+q+r}+\frac{2}{m^{p+q+r}}\right]^{n}\left\|\frac{3+m}{2 m} x\right\|^{p}\left\|\frac{3+m}{2 m} y\right\|^{q}\left\|\frac{3+m}{2 m} z\right\|^{r} \\
& +c\left[\left(\frac{3+m}{2 m}\right)^{p+q+r}+\left(\frac{1+m}{2 m}\right)^{p+q+r}+\frac{2}{m^{p+q+r}}\right]^{n}\left\|\frac{1+m}{2 m} x\right\|^{p}\left\|\frac{1+m}{2 m} y\right\|^{q}\left\|\frac{1+m}{2 m} z\right\|^{r} \\
& +2 c\left[\left(\frac{3+m}{2 m}\right)^{p+q+r}+\left(\frac{1+m}{2 m}\right)^{p+q+r}+\frac{2}{m^{p+q+r}}\right]^{n}\left\|\frac{x}{m}\right\|^{p}\left\|\frac{y}{m}\right\|^{q}\left\|\frac{z}{m}\right\|^{r} \\
& =c\left(\left(\frac{3+m}{2 m}\right)^{p+q+r}+\left(\frac{1+m}{2 m}\right)^{p+q+r}+\frac{2}{m^{p+q+r}}\right)^{n+1}\|x\|^{p}\|y\|^{q}\|z\|^{r}
\end{aligned}
$$

for all $x, y, z \in X \backslash\{0\}$.

Thus, by induction, we have shown that (2.5) holds for all $x, y, z \in$ $X \backslash\{0\}$ and for all $n \in \mathbf{N}$. Letting $n \rightarrow \infty$ in (2.5), we obtain

$$
F_{m}\left(\frac{x+y}{2}+z\right)+F_{m}\left(\frac{x-y}{2}+z\right)=F_{m}(x)+2 F_{m}(z),
$$

for all $x, y, z \in X \backslash\{0\}$. So, we have a sequence $\left(F_{m}\right)_{m \geq m_{0}}$ satisfies (1.3) on $X \backslash\{0\}$ such that

$$
\left\|f(x)-F_{m}(x)\right\| \leq \frac{c\|x\|^{p+q+r}}{m^{r+q}\left(1-\left[\frac{3+m}{2 m}\right]^{p+q+r}-\left[\frac{1+m}{2 m}\right]^{p+q+r}-\frac{2}{m^{p+q+r}}\right)}
$$


for all $x \in X \backslash\{0\}$ and $m \geq m_{0}$. It follows, with $m \rightarrow \infty$, that $f$ is a solution of (1.3) on $X \backslash\{0\}$.

Theorem 2.3. Let $X$ be a normed space, $Y$ be a Banach space, $c \geq 0$ and $p, q, r \in \mathbf{R}$ such that $0<p+q+r<1$. If $f: X \rightarrow Y$ satisfies (2.1) then $f$ is a solution of (1.3) on $X \backslash\{0\}$.

Proof. Since $p+q+r>0$, one of $p, r+q$ must be positive, it is sufficient to consider only the case $p>0$. Replacing $(x, y, z)$ by $\left(\frac{2 x}{m}, 2 x, x\right)$, with $m \in \mathbf{N}_{1}$, in (2.1), we get

$$
\left\|\frac{1}{2} f\left(\frac{1+2 m}{m} x\right)+\frac{1}{2} f\left(\frac{x}{m}\right)-\frac{1}{2} f\left(\frac{2 x}{m}\right)-f(x)\right\| \leq \frac{c 2^{p+q-1}}{m^{p}}\|x\|^{p+q+r}
$$

for all $x \in X \backslash\{0\}$. Define operators $\mathcal{T}: Y^{X \backslash\{0\}} \rightarrow Y^{X \backslash\{0\}}$ and $\Lambda$ : $\mathbf{R}_{+}{ }^{X \backslash\{0\}} \rightarrow \mathbf{R}_{+}{ }^{X \backslash\{0\}}$ by

$$
\begin{aligned}
\mathcal{T} \xi(x) & :=\frac{1}{2} \xi\left(\frac{1+2 m}{m} x\right)+\frac{1}{2} \xi\left(\frac{x}{m}\right)-\frac{1}{2} \xi\left(\frac{2 x}{m}\right), \quad x \in X \backslash\{0\}, \xi \in Y^{X \backslash\{0\}}, \\
\Lambda \delta(x) & :=\frac{1}{2} \delta\left(\frac{1+2 m}{m} x\right)+\frac{1}{2} \delta\left(\frac{x}{m}\right)+\frac{1}{2} \delta\left(\frac{2 x}{m}\right), \quad x \in X \backslash\{0\}, \delta \in \mathbf{R}_{+}{ }^{X \backslash\{0\}} .
\end{aligned}
$$

Then it is easily seen that $\Lambda$ has the form described in (H3) with $k=3$ and

$$
\begin{gathered}
f_{1}(x)=\frac{1+2 m}{m} x, \quad f_{2}(x)=\frac{x}{m}, \quad f_{3}(x)=\frac{2 x}{m}, \quad \text { and } \\
L_{1}(x)=L_{2}(x)=L_{3}(x)=\frac{1}{2}, \quad x \in X \backslash\{0\} .
\end{gathered}
$$

Then the inequality (2.6) take the form

$$
\|\mathcal{T} f(x)-f(x)\| \leq \varepsilon(x), \quad x \in X \backslash\{0\},
$$

with

$$
\varepsilon(x):=\frac{c 2^{p+q-1}}{m^{p}}\|x\|^{p+q+r} .
$$

Moreover, for every $\xi, \mu \in Y^{X \backslash\{0\}}$ and $x \in X \backslash\{0\}$, we have

$$
\begin{aligned}
& \|\mathcal{T} \xi(x)-\mathcal{T} \mu(x)\| \\
& =\frac{1}{2}\left\|(\xi-\mu)\left(f_{1}(x)\right)+(\xi-\mu)\left(f_{2}(x)\right)-(\xi-\mu)\left(f_{3}(x)\right)\right\| \\
& \leq \sum_{i=1}^{3} L_{i}(x)\left\|(\xi-\mu)\left(f_{i}(x)\right)\right\| .
\end{aligned}
$$


So, (H2) is valid. We observe that there exists $m_{0} \in \mathbf{N}_{1}$ such that $\frac{1}{2}\left[\frac{1+2 m}{m}\right]^{p+q+r}+\frac{1}{2}\left[\frac{1}{m}\right]^{p+q+r}+\frac{1}{2}\left(\frac{2}{m}\right)^{p+q+r}<1 \quad$ for all $m \geq m_{0}$.

We have

$$
\begin{aligned}
& \varepsilon^{*}(x):=\sum_{n=0}^{\infty} \Lambda^{n} \varepsilon(x) \\
& =\frac{c 2^{p+q-1}}{m^{p}}\|x\|^{p+q+r} \sum_{n=0}^{\infty}\left(\frac{1}{2}\left[\frac{1+2 m}{m}\right]^{p+q+r}+\frac{1}{2}\left[\frac{1}{m}\right]^{p+q+r}+\frac{1}{2}\left(\frac{2}{m}\right)^{p+q+r}\right)^{n} \\
& =\frac{c 2^{p+q-1}\|x\|^{p+q+r}}{m^{p}\left(1-\frac{1}{2}\left[\frac{1+2 m}{m}\right]^{p+q+r}-\frac{1}{2}\left[\frac{1}{m}\right]^{p+q+r}-\frac{1}{2}\left(\frac{2}{m}\right)^{p+q+r}\right)}
\end{aligned}
$$

for all $x \in X \backslash\{0\}$ and $m \geq m_{0}$.

The rest of the proof is similar to the proof of Theorem 2.2 .

Remark 1. Let $X$ be a normed space, $Y$ be a Banach space, $c \geq 0$ and $p, q, r>0$ such that $p+q+r \neq 1$. If $f: X \rightarrow Y$ satisfies (2.1) on $X$, then $f$ is a solution of (1.3) on $X$.

Theorem 2.4. Let $X$ be a normed space, $Y$ be a Banach space, $c \geq 0$ and $p<0$. If $f: X \rightarrow Y$ satisfies

$$
\left\|f\left(\frac{x+y}{2}+z\right)+f\left(\frac{x-y}{2}+z\right)-f(x)-2 f(z)\right\| \leq c\left(\|x\|^{p}+\|y\|^{p}+\|z\|^{p}\right)
$$

for all $x, y, z \in X \backslash\{0\}$, then $f$ is a solution of (1.3) on $X \backslash\{0\}$.

Proof. Replacing $(x, y, z)$ by $(m x, m x,(1-m) x)$, where $m \in \mathbf{N}_{1}$, in (2.7), we get

$$
\|f((1-m) x)+f(m x)-f(x)\| \leq c\left(2 m^{p}+(m-1)^{p}\right)\|x\|^{p}
$$

for all $x \in X \backslash\{0\}$. Further put 


$$
\begin{gathered}
\mathcal{T} \xi(x)=\xi((1-m) x)+\xi(m x), \quad x \in X \backslash\{0\}, \xi \in Y^{X \backslash\{0\}}, \\
\varepsilon(x):=\left(2 m^{p}+(m-1)^{p}\right)\|x\|^{p} .
\end{gathered}
$$

Inequality (2.8) takes the following form

$$
\|\mathcal{T} f(x)-f(x)\| \leq \varepsilon(x), \quad x \in X \backslash\{0\} .
$$

The operator

$$
\Lambda \delta(x)=\delta((1-m) x)+\delta(m x), \quad x \in X \backslash\{0\}, \delta \in \mathbf{R}_{+}^{X \backslash\{0\}}
$$

has the form described in (H3) with $k=2$ and

$$
f_{1}(x)=(1-m) x, \quad f_{2}(x)=m x, \quad \text { and } \quad L_{1}(x)=L_{2}(x)=1, \quad x \in X \backslash\{0\}
$$

Moreover, for every $\xi, \mu \in Y^{X \backslash\{0\}}$ and $x \in X \backslash\{0\}$, we have

$$
\begin{aligned}
& \|\mathcal{T} \xi(x)-\mathcal{T} \mu(x)\| \\
& =\|(\xi-\mu)((1-m) x)-(\xi-\mu)(m x)\| \\
& \leq \sum_{i=1}^{2} L_{i}(x)\left\|(\xi-\mu)\left(f_{i}(x)\right)\right\| .
\end{aligned}
$$

So, (H2) is valid. Now, we can find $m_{0} \in \mathbf{N}_{1}$ such that

$$
(m-1)^{p}+m^{p}<1 \text { for all } m \geq m_{0} .
$$

Therefore, we obtain that

$$
\begin{aligned}
& \varepsilon^{*}(x):=\sum_{n=0}^{\infty} \Lambda^{n} \varepsilon(x) \\
& =\frac{c\left(2 m^{p}+(m-1)^{p}\right)\|x\|^{p}}{1-(m-1)^{p}-m^{p}}
\end{aligned}
$$

for all $x \in X \backslash\{0\}$ and $m \geq m_{0}$. The rest of the proof is similar to the proof of Theorem 2.1 .

Corollary 2.4.1. Let $X$ be a normed space and $Y$ be a Banach space. Let $H: X^{3} \rightarrow Y$ be a mapping such that $H\left(x_{0}, y_{0}, z_{0}\right) \neq 0$ for some $x_{0}, y_{0}, z_{0} \in X$ and

$$
\|H(x, y, z)\| \leq c\|x\|^{p}\|y\|^{q}\|z\|^{r},
$$

or 


$$
\|H(x, y, z)\| \leq c\left(\|x\|^{p}+\|y\|^{p}+\|z\|^{p}\right),
$$

for all $x, y, z \in X \backslash\{0\}$, where $c \geq 0$ and $p, q, r \in \mathbf{R}$. Assume that the numbers $p, q, r$ satisfy one of the following conditions:

$$
\text { (i) } p+q+r<0 \quad \text { (ii) } 0<p+q+r<1 \quad \text { (iii) } p+q+r>1
$$

in the case (2.9) and

$$
\text { (iv) } p<0 \text { in the case }(2.10)
$$

Then the functional equation

$h\left(\frac{x+y}{2}+z\right)+h\left(\frac{x-y}{2}+z\right)=H(x, y, z)+h(x)+2 h(z), \quad x, y, z \in X \backslash\{0\}$

has no solution in the class of functions $h: X \rightarrow Y$.

Proof. Suppose that $h: X \rightarrow Y$ is a solution to (2.11). Then (2.1) or (2.7) holds, and consequently, according to the above theorems, $h$ is Jensen on $X \backslash\{0\}$, which means that $H\left(x_{0}, y_{0}, z_{0}\right)=0$. This is a contradiction.

Remark 2. Let $X$ be a normed space and $Y$ be a Banach space. Let $H$ : $X^{3} \rightarrow Y$ be a mapping such that $H\left(x_{0}, y_{0}, z_{0}\right) \neq 0$ for some $x_{0}, y_{0}, z_{0} \in X$ and

$$
\|H(x, y, z)\| \leq c\|x\|^{p}\|y\|^{q}\|z\|^{r},
$$

for all $x, y, z \in X$, where $c \geq 0$ and $p, q, r>0$ with $p+q+r \neq 1$.

Then the functional equation

$$
h\left(\frac{x+y}{2}+z\right)+h\left(\frac{x-y}{2}+z\right)=H(x, y, z)+h(x)+2 h(z), \quad x, y, z \in X
$$

has no solution in the class of functions $h: X \rightarrow Y$. 


\section{References}

[1] T. Aoki, On the stability of the linear transformation in Banach spaces, J. Math. Soc. Japan, 2, pp. 64-66, (1950).

[2] C. Baak, Cauchy-Rassias stability of Cauchy-Jensen additive mappings in Banach spaces, Acta Mathematica Sinica, English Series, Vol.22, No. 6, pp. 1789-1796, (2006).

[3] A. Bahyrycz, M. Piszczek, Hyperstability of the Jensen functional equation, Acta Math. Hungar. 142 (2), pp. 353-365, (2014).

[4] D. G. Bourgin, Approximately isometric and multiplicative transformations on continuous function rings, Duke Math. J., 16, pp. 385-397, (1949).

[5] D. G. Bourgin, Classes of transformations and bordering transformations, Bull. Amer. Math. Soc., 57, pp. 223-237, (1951).

[6] J. Brzdek, J. Chudziak, Z. Páles, A fixed point approach to stability of functional equations, Nonlinear Anal., vol. 74, no. 17, pp. 6728-6732, (2011).

[7] J. Brzdek, Remarks on hyperstability of the the Cauchy equation, Aequations Mathematicae, 86, pp. 255-267, (2013).

[8] J. Brzdek, Hyperstability of the Cauchy equation on restricted domains, Acta Math. Hungarica, 141 (1-2), pp. 58-67, (2013).

[9] J. Brzdek, A hyperstability result for the Cauchy equation. Bulletin of the Australian Mathematical Society 89, pp. 33-40, (2014).

[10] L. Cădariu, V. Radu, Fixed points and the stability of Jensens functional equation, Journal of Inequalities in Pure and Applied Mathematics, 4(1), (2003).

[11] Z. Gajda, On stability of additive mappings, Int. J. Math. Math. Sci. 14, pp. 431-434, (1991).

[12] M. E. Gordji, H. Khodaei, M. Kamyar, Stability of Cauchy-Jensen type functional equation in generalized fuzzy normed spaces, Computers and Mathematics with Applications, 62, pp. 2950-2960, (2011). 
[13] E. Gselmann, Hyperstability of a functional equation, Acta Mathematica Hungarica, vol. 124, no. 1-2, pp. 179-188, (2009).

[14] D. H. Hyers, On the stability of the linear functional equation, Proc. Nat. Acad. Sci., U.S.A., 27, pp. 222-224, (1941).

[15] K. W. Jun, H. M. Kim, J. M. Rassias, Extended Hyers-Ulam stability for Cauchy-Jensen mappings, J. Diference Equ. Appl., 13(12), pp. 1139-1153, (2007).

[16] S. M. Jung, M. S. Moslehian, P. K. Sahoo, Stability of a generalized Jensen equation on restricted domains, J. Math. Ineq., 4, pp. 191-206, (2010).

[17] Z. Kominek, On a local stability of the Jensen functional equation, Demonstratio Math. 22, pp. 499-507, (1989).

[18] Y. H. Lee, K. W. Jun, A generalization of the Hyers-Ulam-Rassias stability of Jensens equation, J. Math. Anal. Appl., 238, pp. 305-315, (1999).

[19] G. Maksa, Z. Páles, Hyperstability of a class of linear functional equations, Acta Math., vol. 17, no. 2, pp. 107-112, (2001).

[20] C. Park, Fixed points and Hyers-Ulam-Rassias stability of CauchyJensen functional equations in Banach algebras, Fixed. Point. Theory. Appl., 15, (2007) : Article ID 50175.

[21] C. Park, J. M Rassias, Stability of the Jensen-type functional equation in $C^{*}$-algebras: A fixed point approach. Abstract and Applied Analysis, Volume 2009 (2009), Article ID 360432, 17 pages.

[22] M. Piszczek, Remark on hyperstability of the general linear equation, Aequations Mathematicae, (2013).

[23] Th. M. Rassias, On the stability of the linear mapping in Banach spaces, Proc. Amer. Math. Soc., 72, pp. 297-300, (1978).

[24] Th. M. Rassias, On a modified HyersUlam sequence, J. Math. Anal. Appl. 158, pp. 106-113, (1991).

[25] Th. M. Rassias and P. Semrl, On the behavior of mappings which do not satisfy Hyers-Ulam stability, Proc. Amer. Math. Soc. 114, pp. 989-993, (1992). 
[26] J. M. Rassias, On the Ulam stability of Jensen and Jensen type mappings on restricted domains, J. Math. Anal. Appl. 281, pp. 516-524, (2003).

[27] S. M. Ulam, Problems in Modern Mathematics, Chapter IV, Science Editions, Wiley, New York, (1960).

\section{Iz-iddine EL-Fassi}

Department of Mathematics,

Faculty of Sciences,

University of Ibn Tofail,

Kenitra,

Morocco

e-mail : izidd-math@hotmail.fr

and

\section{Samir Kabbaj}

Department of Mathematics,

Faculty of Sciences,

University of Ibn Tofail,

Kenitra,

Morocco

e-mail: samkabbaj@yahoo.fr 